International Journal of Foundations of Computer Science

Vol. 21, No. 6 (2010) 1101-1105

(C) World Scientific Publishing Company

DOI: $10.1142 /$ S0129054110007763

\title{
AUTHOR INDEX \\ VOLUME 21 (2010)
}

Ada, A., On the non-deterministic communication complexity of regular languages

21 (2010) 479-493

Adamaszek, A., Czumaj, A. and Lingas, A., PTAS for $k$-tour cover problem on the plane for moderately large values of $k$

Afonin, S. and Khazova, E., On the structure of finitely generated semigroups of unary regular languages

Ando, E., Ono, H., Sadakane, K. and Yamashita, M., The space complexity of leader election in anonymous networks

Apostolico, A. and Tagliacollo, C., Optimal extraction of irredundant motif bases

Atanasiu, A., Parikh matrices, amiability and istrail morphism

Atanasiu, R.-F., Parikh matrix mapping and languages

Aytac, A. and Odabas, Z. N., Computing the rupture degree in composite graphs

Bassino, F., Giambruno, L. and Nicaud, C., The average state complexity of rational operations on finite languages

Bell, P. C. and Potapov, I., On the undecidability of the identity correspondence problem and its applications for word and matrix semigroups

Blanchet-Sadri, F., Oey, T. and Rankin, T. D., Fine and Wilf's theorem for partial words with arbitrarily many weak periods

Bruda, S. D. and Zhang, Y., Collapsing the hierarchy of parallel computational models

Chang, C.-L. and Lyuu, Y.-D., Efficient testing of forecasts

Chang, C.-L., see Ti, Y.-W.

Chang, J.-M., see Yang, J.-S.

Chang, R.-X., see Lee, C. C.

Chao, K. M., see Hsu, P. H.

Chen, K. Y., see Hsu, P. H.

Chen, L., see Xu, M.

Chin, F. Y. L., Ting, H.-F. and Zhang, Y., One-space bounded algorithms for two-dimensional bin packing

Chunguang, Z., see Junping, Z.

Crochemore, M., Fazekas, S. Z., Iliopoulos, C. S. and Jayasekera, I., Number of occurrences of powers in strings

21 (2010) 893-904

21 (2010) 689-704

21 (2010) 427-440

21 (2010) 1035-1047

21 (2010) 1021-1033

21 (2010) 993-1004

21 (2010) 311-319

21 (2010) 495-516

21 (2010) 963-978

21 (2010) 705-722

21 (2010) 441-457

21 (2010) 61-72

21 (2010) 321-327

21 (2010) 73-90

21 (2010) 979-991

21 (2010) 925-939

21 (2010) 925-939

21 (2010) 1005-1019

21 (2010) 875-891

21 (2010) 1073-1088

21 (2010) 535-547 
Csuhaj-Varjú, E., Dassow, J. and Vaszil, G., Variants of competence-based derivations in $\mathrm{CD}$ grammar systems

Czumaj, A., see Adamaszek, A.

Dassow, J., Stiebe, R. and Truthe, B., Generative capacity of subregularly tree controlled grammars

Dassow, J., see Csuhaj-Varjú, E.

Ehrenfeucht, A., Main, M. and Rozenberg, G., Combinatorics of life and death for reaction systems

Elkind, E., Genest, B., Peled, D. and Spoletini, P., Quantifying the discord: Order discrepancies in message sequence charts

Fazekas, S. Z., see Crochemore, M.

Filiot, E., Talbot, J.-M. and Tison, S., Tree automata with global constraints

Forsell, M., On the performance and cost of some PRAM models on CMP hardware

Friedmann, O., The Stevens-Stirling-algorithm for solving parity games locally requires exponential time

Gawrychowski, P., Krieger, D., Rampersad, N. and Shallit, J., Finding the growth rate of a regular or context-free language in polynomial time

Geeraerts, G., Raskin, J.-F. and Van Begin, L., On the efficient computation of the minimal coverability set of petri nets

Genest, B., see Elkind, E.

Giambruno, L., see Bassino, F.

Glöckler, J., A taxonomy of deterministic forgetting automata

$\mathrm{Gu}, \mathrm{D}$., see Ren, Y.

Haddad, S., see Recalde, L.

Holub, Š. and Nowotka, D., On the relation between periodicity and unbordered factors of finite words

Hsu, P. H., Chen, K. Y. and Chao, K. M., Finding all approximate gapped palindromes

Huang, G.-D. and Wang, B.-Y., Complete SAT-based model checking for context-free processes

Huang, K.-S., see Peng, Y.-H.

Iliopoulos, C. S., see Crochemore, M.

Ito, Y. and Nakano, K., Low-latency connected component labeling using an FPGA

Jansen, K. and Solis-Oba, R., Approximation schemes for scheduling jobs with chain precedence constraints

Jayasekera, I., see Crochemore, M.

Jones, K., see Little, S.

Junping, Z., Ping, H., Minghao, Y. and Chunguang, Z., Phase transitions of EXPSPACE-complete problems

Kakugawa, H., see Kamei, S.

Kamei, S. and Kakugawa, H., A self-stabilizing distributed approximation algorithm for the minimum connected dominating set

21 (2010) 549-569

21 (2010) 893-904

21 (2010) 723-740

21 (2010) 549-569

21 (2010) 345-356

21 (2010) 211-233

21 (2010) 535-547

21 (2010) 571-596

21 (2010) 387-404

21 (2010) 277-287

21 (2010) 597-618

21 (2010) 135-165

21 (2010) 211-233

21 (2010) 495-516

21 (2010) 619-631

21 (2010) 1049-1063

21 (2010) 235-256

21 (2010) 633-645

21 (2010) 925-939

21 (2010) 115-134

21 (2010) 51-59

21 (2010) 535-547

21 (2010) 405-425

21 (2010) 27-49

21 (2010) 535-547

21 (2010) 191-210

21 (2010) 1073-1088

21 (2010) 459-476

21 (2010) 459-476 
Kaminski, M. and Zeitlin, D., Finite-memory automata with non-deterministic reassignment

Karpiński, M., Ruciński, A. and Szymańska, E., Computational complexity of the perfect matching problem in hypergraphs with subcritical density

21 (2010) 905-924

Kärki, T., A new proof of the three-squares lemma for partial words with one hole

Kellerer, H. and Strusevich, V. A., Minimizing total weighted earliness-tardiness on a single machine around a small common due date: An FPTAS using quadratic knapsack

Khazova, E., see Afonin, S.

Klíma, O. and Polák, L., Hierarchies of piecewise testable languages

Klíma, O. and Polák, L., Literally idempotent languages and their varieties - two letter case

Ko, H.-J., see Lee, C. C.

Krieger, D., see Gawrychowski, P.

Kundeti, V. and Rajasekaran, S., On the hardness of the border length minimization problem on a rectangular array

Kupferman, O. and Lustig, Y., Latticed simulation relations and games

Kutrib, M., Messerschmidt, H. and Otto, F., On stateless two-pushdown automata and restarting automata

Leangsuksun, C., see Paun, M.

Lee, C. C., Chang, R.-X. and Ko, H.-J., Improving two novel three-party encrypted key exchange protocols with perfect forward secrecy

Lehtinen, T. and Okhotin, A., Boolean grammars and gsm mappings

Li, Z.-B., see Xu, M.

Lingas, A., see Adamaszek, A.

Little, S., Walter, D., Jones, K., Myers, C. and Sen, A., Analog/mixed-signal circuit verification using models generated from simulation traces

21 (2010) 1065-1071

21 (2010) 357-383

21 (2010) 689-704

21 (2010) 517-533

21 (2010) 761-780

21 (2010) 979-991

21 (2010) 597-618

21 (2010) 1089-1100

21 (2010) 167-189

21 (2010) 781-798

21 (2010) 329-344

21 (2010) 979-991

21 (2010) 799-815

21 (2010) 1005-1019

21 (2010) 893-904

21 (2010) 191-210

Lohrey, M., Compressed membership problems for regular expressions and hierarchical automata

Lustig, Y., see Kupferman, O.

Lyuu, Y.-D., see Chang, C.-L.

Lyuu, Y.-D., see Ti, Y.-W.

Main, M., see Ehrenfeucht, A.

Malcher, A., Mereghetti, C. and Palano, B., Sublinearly space bounded iterative arrays

Maletti, A. and Tîrnăucă, C. I., Properties of quasi-relabeling tree bimorphisms

Manea, F., Mitrana, V. and Yokomori, T., Some remarks on the hairpin completion

21 (2010) 817-841

21 (2010) 167-189

21 (2010) 61-72

21 (2010) 321-327

21 (2010) 345-356

21 (2010) 843-858

21 (2010) 257-276

Mantaci, R., Mantaci, S. and Restivo, A., Balance properties and distribution of squares in circular words

21 (2010) 859-872

21 (2010) 647-664 
Mantaci, S., see Mantaci, R.

21 (2010) 647-664

Mereghetti, C., see Malcher, A.

21 (2010) 843-858

Messerschmidt, H., see Kutrib, M.

21 (2010) 781-798

Minghao, Y., see Junping, Z.

21 (2010) 1073-1088

Mitrana, V., see Manea, F.

21 (2010) 859-872

Morin, R., Unambiguous shared-memory systems

Moriya, E. and Otto, F., On alternating phrase-structure

21 (2010) 665-685 grammars

Myers, C., see Little, S.

21 (2010) 1-25

21 (2010) 191-210

Nakano, K., see Ito, Y.

Naksinehaboon, N., see Paun, M.

21 (2010) 405-425

21 (2010) 329-344

Nassar, R., see Paun, M.

21 (2010) 329-344

Nicaud, C., see Bassino, F.

Nowotka, D., see S̆. Holub

21 (2010) 495-516

21 (2010) 633-645

Odabas, Z. N., see Aytac, A.

21 (2010) 311-319

Oey, T., see Blanchet-Sadri, F.

21 (2010) 705-722

Okhotin, A., see Lehtinen, T.

Ono, H., see Ando, E.

Otto, F., see Kutrib, M.

Otto, F., see Moriya, E.

Palano, B., see Malcher, A.

Paun, M., Naksinehaboon, N., Nassar, R., Leangsuksun, C., Scott, S. L. and Taerat, N., Incremental checkpoint schemes for Weibull failure distribution

$21(2010) 799-815$

21 (2010) 427-440

21 (2010) 781-798

21 (2010) $1-25$

21 (2010) 843-858

Peled, D., see Elkind, E.

Peng, Y.-H., Yang, C.-B., Tseng, K.-T. and Huang, K.-S., An algorithm and applications to sequence alignment with weighted constraints

Ping, H., see Junping, Z.

Polák, L., see Klíma, O.

Polák, L., see Klíma, O.

Potapov, I., see Bell, P. C.

Rajasekaran, S., see Kundeti, V.

Rampersad, N., see Gawrychowski, P.

Rankin, T. D., see Blanchet-Sadri, F.

Raskin, J.-F., see Geeraerts, G.

Recalde, L., Haddad, S. and Silva, M., Continuous petri nets: Expressive power and decidability issues

Ren, Y., Gu, D., Wang, S. and Zhang, X., Hierarchical identity-based proxy re-encryption without random oracles

Restivo, A., see Mantaci, R.

Rozenberg, G., see Ehrenfeucht, A.

Ruciński, A., see Karpiński, M.

Sadakane, K., see Ando, E.

Salomaa, A. and Yu, S., Subword occurrences, Parikh matrices and Lyndon images

21 (2010) 329-344

21 (2010) 211-233

21 (2010) 51-59

21 (2010) 1073-1088

21 (2010) 517-533

21 (2010) 761-780

21 (2010) 963-978

21 (2010) 1089-1100

21 (2010) 597-618

21 (2010) 705-722

21 (2010) 135-165

21 (2010) 235-256

21 (2010) 1049-1063

21 (2010) 647-664

21 (2010) 345-356

21 (2010) 905-924

21 (2010) 427-440

21 (2010) 91-111 
Schnoor, H., The complexity of model checking for Boolean formulas
21 (2010) 289-309
21 (2010) 329-344
21 (2010) 191-210
21 (2010) 597-618
21 (2010) 321-327
21 (2010) 235-256
21 (2010) 27-49
21 (2010) 211-233
21 (2010) 723-740
21 (2010) 357-383
21 (2010) 905-924
21 (2010) 329-344
21 (2010) 1035-1047
21 (2010) 571-596
21 (2010) 73-90

Scott, S. L., see Paun, M.

Sen, A., see Little, S.

Shallit, J., see Gawrychowski, P.

Shen, A., see Ti, Y.-W.

Silva, M., see Recalde, L.

Solis-Oba, R., see Jansen, K.

Spoletini, P., see Elkind, E.

Stiebe, R., see Dassow, J.

Strusevich, V. A., see Kellerer, H.

Szymańska, E., see Karpiński, M.

Taerat, N., see Paun, M.

Tagliacollo, C., see Apostolico, A.

Talbot, J.-M., see Filiot, E.

Tang, S.-M., see Yang, J.-S.

Ti, Y.-W., Chang, C.-L., Lyuu, Y.-D. and Shen, A., Sets of $K$-independent strings

Ting, H.-F., see Chin, F. Y. L.

Tîrnăucă, C. I., see Maletti, A.

Tison, S., see Filiot, E.

Truthe, B., see Dassow, J.

Tseng, K.-T., see Peng, Y.-H.

Van Begin, L., see Geeraerts, G.

Vaszil, G., see Csuhaj-Varjú, E.

Walter, D., see Little, S.

Wang, B.-Y., see Huang, G.-D.

Wang, S., see Ren, Y.

Wang, Y.-L., see Yang, J.-S.

$\mathrm{Xu}$, M., Chen, L., Zeng, Z. and Li, Z.-B., Termination analysis of linear loops

21 (2010) 321-327

21 (2010) 875-891

21 (2010) 257-276

21 (2010) 571-596

21 (2010) 723-740

21 (2010) 51-59

21 (2010) 135-165

21 (2010) 549-569

21 (2010) 191-210

21 (2010) 115-134

21 (2010) 1049-1063

21 (2010) 73-90

21 (2010) 1005-1019

Yamakami, T., The roles of advice to one-tape linear-time Turing machines and finite automata

Yamashita, M., see Ando, E.

Yang, C.-B., see Peng, Y.-H.

Yang, J.-S., Chang, J.-M., Tang, S.-M. and Wang, Y.-L., Constructing multiple independent spanning trees on recursive circulant graphs $G\left(2^{m}, 2\right)$

Yokomori, T., see Manea, F.

Yu, S., see Salomaa, A.

Zeitlin, D., see Kaminski, M.

Zeng, Z., see $\mathrm{Xu}, \mathrm{M}$.

Zhang, X., see Ren, Y.

Zhang, Y., see Bruda, S. D.

Zhang, Y., see Chin, F. Y. L.

21 (2010) 941-962

21 (2010) 427-440

21 (2010) 51-59

21 (2010) 73-90

21 (2010) 859-872

21 (2010) 91-111

21 (2010) 741-760

21 (2010) 1005-1019

21 (2010) 1049-1063

21 (2010) 441-457

21 (2010) 875-891 